(c) 2018 This paper is a postprint of a paper submitted to and accepted for publication in the Proceedings of the 12th European Conference on Antennas and Propagation (EuCAP 2018) and is subject to Institution of Engineering and Technology Copyright. The copy of record is available at the IET Digital Library DOI: $10.1049 /$ cp. 2018.0552 


\title{
A Complex Low-RCS Antenna System for the Placement of mm-Wave Automotive Radars behind Car Bumpers
}

\author{
Claudia Vasanelli, Frank Bögelsack, and Christian Waldschmidt, \\ Ulm University, Institute of Microwave Engineering, 89081 Ulm, Germany \\ \{claudia.vasanelli, frank.boegelsack, christian.waldschmidt\}@uni-ulm.de
}

\begin{abstract}
Automotive radars are one of the key sensor technologies to develop reliable fully-automated cars. They are usually installed behind the bumper of the vehicle and therefore, multiple reflections can occur. The multiple reflections can be mitigated with the use of low-RCS (radar cross-section) antenna arrays. This paper presents the design of a complex low-RCS multiple antenna system that mimics a typical MIMO (multiple-input multiple-output) configuration. A prototype has been developed and tested. Approximately $17 \mathrm{~dB}$ of RCS reduction has been experimentally achieved. The measurement results show that the use of such a low-RCS antenna system can successfully reduce the amplitude of multiple reflections.
\end{abstract}

Index Terms-radar cross-sections, microstrip antenna arrays, radar antennas, millimeter wave radar.

\section{INTRODUCTION}

Car manufacturers are putting tremendous effort into developing reliable highly-automated or fully-automated vehicles. To achieve this task a number of sensors, like lidar, radar, or cameras, are used to sense the surrounding environment. Among them radar sensors offer unique features, mainly due to their robustness against harsh environmental conditions [1].

Automotive radars operate in the frequency range between $76 \mathrm{GHz}$ and $81 \mathrm{GHz}$ [2]. Thanks to the small wavelength it is possible to realize compact sensors, that could be easily integrated in a car. Typically, the radars are placed behind the front and rear bumper.

The bumpers are complex multilayer structures made of low-cost plastic materials. These layers can show significant different values of permittivity, ranging from 2 to 8 [3], while the coating contains often metal particles. A bumper can thus strongly reflect an impinging electromagnetic wave.

Let consider the following example. A radar sensor illuminates a target on the street. The wave reflected back by the target will impinge on the housing and on the printed circuit board (PCB) of the sensor, which will in turn reflect the impinging wave towards the bumper. The bumper will also reflect a part of the impinging wave and finally the antennas of the radar will measure the signal of interest from the target superimposed on the multiple reflections between radar and bumper. Both signals will be detected in the same range cell. The multiple reflections can potentially lead to a wrong estimation of the position of the target or angular ambiguities [4]. The investigation of possible techniques to mitigate the multiple reflections between the radar and the car fascia is thus of great practical interest.

Some previous works [5], [6] explained that a possible approach to reduce the multiple reflections can be the design of a radar sensor with low-RCS, i.e. to develop a sensor that scatters less power back in boresight direction when it is illuminated by an impinging wave. This solution is indeed quite appealing, since it is totally independent from the actual geometry and material composition of the bumper.

The low-RCS antenna system proposed in this work is based on the principle of operation of the surface with low RCS presented in [7], [8], that has been tested at mm-wave frequencies in [5]. The work described in [6] shows the design of a series-fed microstrip array with low RCS. This paper generalizes for the first time the design to a complex multiple antenna system that mimics the typical antenna setup of a mm-wave automotive radar.

The paper is organized as follows. First, Section II recalls briefly the principle of the low-RCS antenna. Section III presents in detail the measurement setup and gives the details of the design and of the experimental results of the proposed antenna system. Section IV draws finally the conclusions.

\section{PRinciple of Operation}

To understand the principle of operation of the low-RCS antenna systems presented in this paper it is worth to briefly describe the low-RCS chessboard-like surface originally presented in [7] and then further developed in [5], [8]. The surface unit cell consists of a periodic displacement of AMC and PEC (perfect electric conductor). It is well known that at its resonance frequency an AMC has a reflection coefficient of +1 [7] and it is thus $180^{\circ}$ out of phase compared to a PEC plate. If a plane wave impinges on the surface, a destructive interference will take place in the boresight direction and the energy will be redirected in different directions. Since a full-description of the chessboard-like surface falls outside the purpose of this work, the reader can refer to [5], [8], [7] for additional details.

The same basic principle can be applied for the development of a low-RCS antenna. An AMC can be placed around a planar antenna in order to achieve destructive interference in the wave reflected back. Following this method, in [6] a seriesfed microstrip patch array, that operates at $77 \mathrm{GHz}$, has been 
surrounded by a set of square patches that acts as AMC. The results presented in [6] demonstrated that with the proposed design it is possible to successfully achieve $20 \mathrm{~dB}$ of RCS reduction.

An interesting feature of this approach is that the fabrication complexity of the low-RCS antenna is not higher than the one of a standard patch antenna array. Indeed, the AMC can be realized with the same fabrication process of the array and it can be placed easily on the same PCB layer. Therefore, using such a low-RCS array does not increase in the end the complexity of the radar sensor. Finally, the use of the AMC patches does not affect the performance of the antenna. Their presence does not cause any significant impact neither in the impedance matching nor in the radiation pattern. The reference [6] gives more details about this point

For the first time, this paper generalizes the aforementioned approach towards the design of a complex multiple antenna system with low-RCS. The focus is set on MIMO configurations, which are gaining more and more importance in $\mathrm{mm}$ wave automotive radar sensors, due to their ability to improve the angular resolution of the system by keeping compact the dimensions of the actual antenna aperture [9].

The reflection from a low-RCS antenna system can be written as

$$
r=10 \log \left|w_{\mathrm{AMC}} A_{\mathrm{AMC}} \mathrm{e}^{\mathrm{j} \varphi_{\mathrm{AMC}}}+w_{\text {ant }} A_{\text {ant }} \mathrm{e}^{\mathrm{j} \varphi_{\text {ant }}}\right|^{2} \mathrm{~dB},
$$

where $A_{\mathrm{AMC}}$ and $A_{\mathrm{ant}}$ are the amplitudes of the reflection coefficients of the AMC and the antenna array, $\varphi_{\text {AMC }}$ and $\varphi_{\text {ant }}$ are the respective phases, and $w_{\mathrm{AMC}}$ and $w_{\text {ant }}$ are the weights assigned to the AMC and to the antenna, which depend on their area occupation on the PCB. As it can be understood from this simple formula, if the amplitude and phase of the wave reflected by the antenna in a specific direction are known, then it is possible to design a proper AMC so that destructive interference can be achieved. The amplitude of the RCS reduction calculated with (1) is thus a weighted average of the contribution of the antenna and of the AMC.

The fundamental requisite for designing a low-RCS antenna is the knowledge of the amplitude and phase of the wave reflected back by the antenna. In general, the backscattering or the RCS of an antenna can be described by a structural mode and an antenna mode [10]. The structural mode depends only on the antenna shape and material, i.e. on its structure; on the other hand, the antenna mode is directly related to the antenna impedance matching, and it has the same pattern as the radiation pattern of the antenna. Previous studies [11], [12] showed that the antenna mode of microstrip antennas is negligible compared to the structural mode. More recent studies [6] verified this property at mm-wave frequencies, too. For this reason, this work does not take into account the actual matching of the antenna for the development of the low-RCS counterpart.

Since microstrip arrays are often employed for automotive radar applications [2], this work focuses only on this kind of antennas.

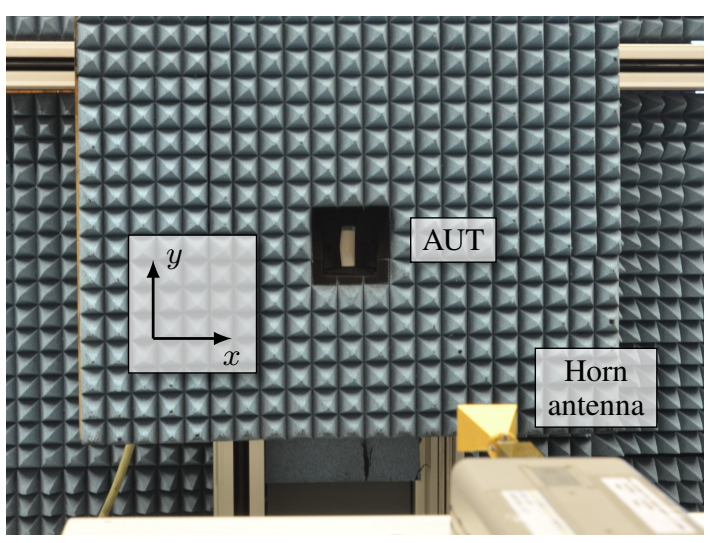

Fig. 1. Measurement setup for the characterization of the backscattering from the antenna in the normal incidence case.

\section{DESIGN AND CHARACTERIZATION OF LOW-RCS ANTENNA ARRAYS}

This section presents the design and the experimental characterization of the microstrip low-RCS multiple antenna system.

All the structures presented in this work have been realized on a single layer substrate of RO3003 from Rogers Corporation with $127 \mu \mathrm{m}$ thickness and a nominal value of permittivity $\varepsilon_{r}=3$. The structures have been realized with standard photoetching process and the visual inspection of the prototypes showed that the etching tolerances are roughly equal to $15 \mu \mathrm{m}$.

\section{A. Measurement Setup}

Before presenting the details of the designs and the measurement results, it is convenient to briefly describe the experimental setup used in this work to measure the RCS reduction in the normal incidence case, as shown in Fig. 1. As it can be noticed, it is a monostatic measurement setup where a 25-dBi standard-gain horn antenna illuminates the antenna under test (AUT). The distance between the horn antenna and the AUT is approximately $1.4 \mathrm{~m}$. By means of time gating it is possible to filter out the unwanted contributions and to collect only the response from the AUT. Since the horn antenna covers the complete E-band $(60 \mathrm{GHz}-90 \mathrm{GHz})$, the setup offers enough spatial resolution to isolate the contribution of the AUT.

The AUT is fixed to a plastic mount, which is in turn controlled by a stepped motor, that allows to accurately set its position in $x$ - and $y$-direction. The measurement results are quite sensitive to alignment errors. To mitigate them, a set of 25 measurements has been collected on a rectangular planar scanning area. The final results is then calculated as average of the 25 different contributions.

As a matter of course, it is of high interest to the test the properties of the low-RCS antennas in a realistic scenario, namely behind the bumper of a car. This can be simply done by placing a sample of typical bumper material between the horn antenna and the AUT with the setup depicted in Fig. 2. 


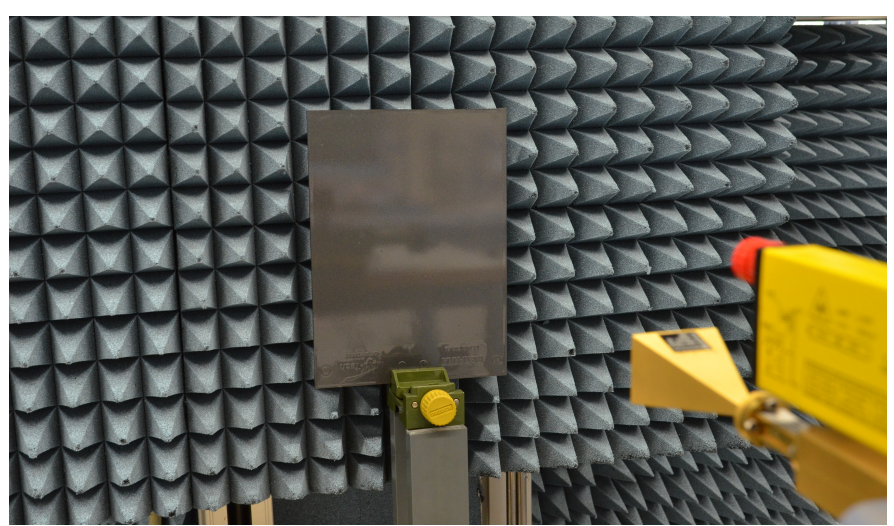

Fig. 2. Measurement setup to characterize the multiple reflections between bumper and AUT. A bumper sample has been placed between the AUT and the horn antenna.

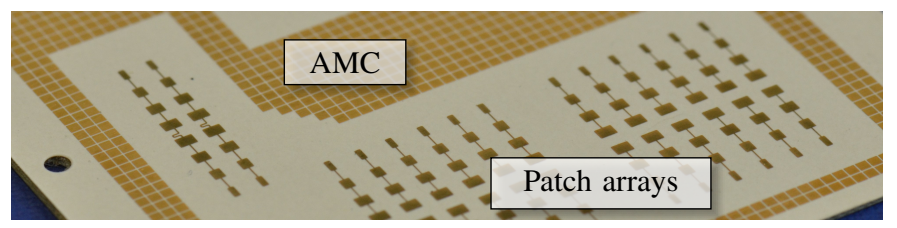

Fig. 3. Photo of the fabricated antenna system composed of series-fed microstrip arrays integrated with the AMC. The external dimensions of the PCBs are $63 \mathrm{~mm} \times 67 \mathrm{~mm}$

The here used bumper sample is $2.8 \mathrm{~mm}$ thick and it is metallic silver painted. The distance between the sample and the AUT is approximately $13 \mathrm{~cm}$, while the distance between the horn antenna and the AUT is about $64 \mathrm{~cm}$. In such a setup, the horn antenna mimics the signal that would be reflected back by a target in the scenario described in the Section I.

\section{B. Multiple Antenna System}

Figure 3 presents the prototype of a multiple antenna system integrated with the AMC. The AMC is composed of a set of square patches; the edge length is $0.87 \mathrm{~mm}$ and the gap between adjacent patches is $0.13 \mathrm{~mm}$. The antenna configuration is a typical example of a mm-wave MIMO radar configuration. As it can be noticed, the feeding network has been neglected.

Due to the large dimensions of the structure, full-wave simulations are not feasible for the development of the lowRCS array configuration. To overcome this limitation, an empirical design approach has been used. The method relies on (1). First, the reflection coefficient of the antenna without the AMC, described by the terms $A_{\text {ant }}$ and $\varphi_{\text {ant }}$, can be obtained by means of measurements. Once these values are known, the proper dimensions of the AMC can be calculated with fullwave simulations, and finally, by changing the weights $w_{\mathrm{AMC}}$ and $w_{\text {ant }}$, the RCS-reduction can be further adjusted. For the prototype of Fig. 3, the weight $w_{\text {AMC }}$ has been set to 0.4 and $w_{\text {ant }}$ to 0.6 .

It must be observed that the empirical method proposed in this paper does not provide any rigorous solution to the problem of designing a complex low-RCS antenna system. In

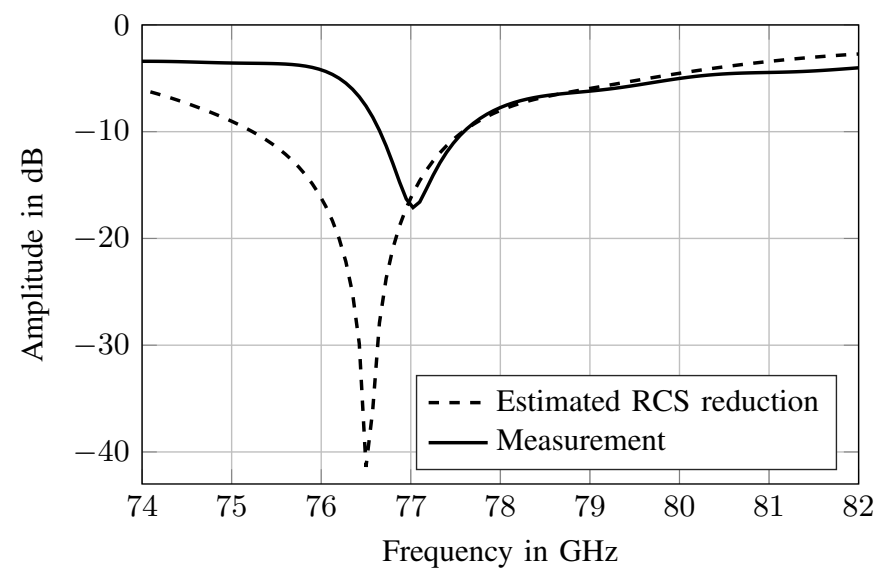

Fig. 4. Estimated, using (1), and measured backscattering from the structure of Fig. 3. The solid curve has been normalized to the measurement results of an antenna system with the same dimension of the prototype of Fig. 3, but without the integration of the AMC.

particular, the main limitation of this approach is related to the use of phase measurement results to get $\varphi_{\text {ant }}$ in (1). At mm-wave frequencies it is well-known that the accuracy of phase measurements is limited. However, as the next results will show, this empirical procedure is still a valuable support where no analytical or numerical solutions are available.

Figure 4 presents the measured RCS reduction in the boresight direction. At $77 \mathrm{GHz}$ the RCS reduction is around $17 \mathrm{~dB}$. The plot shows also the estimated value of the RCS reduction according to (1). It can be noticed that the estimated values fairly agree with the measurement results.

The prototypes have been characterized in the oblique incidence case, too. For this purpose, the AUT has been placed on a turntable in an anechoic chamber in order to take the measurement while performing a sweep on the azimuth plane. The results are presented in Figs. 5a and $5 \mathrm{~b}$ at $77 \mathrm{GHz}$ and $79 \mathrm{GHz}$, respectively. As it can be seen, the backscattering from the prototype of Fig. 3 has been compared to the one of a similar antenna system without the integration of the AMC. The results confirm again that it is possible to achieve a significant RCS reduction in the boresight direction (angle of $0^{\circ}$ ); moreover, the curves show that at $77 \mathrm{GHz}$ the structure with the AMC has a lower backscattering compared to that one without the $\mathrm{AMC}$ within $\pm 10^{\circ}$. Outside this range the amplitude becomes comparable or even larger than in the standard case without AMC. This is however not surprising, since the incident power is simply reflected back in different directions; this kind of behavior is similar to the properties already observed for the low-RCS chessboard surface [5]. Similar conclusions can be drawn for the results at $79 \mathrm{GHz}$ presented in Fig. 5b.

Finally, the fabricated antenna systems, with and without AMC, have been tested behind the car bumper sample with the experimental setup described in Fig. 2. The results are depicted in Fig. 6 and they have been plotted in the time domain to 


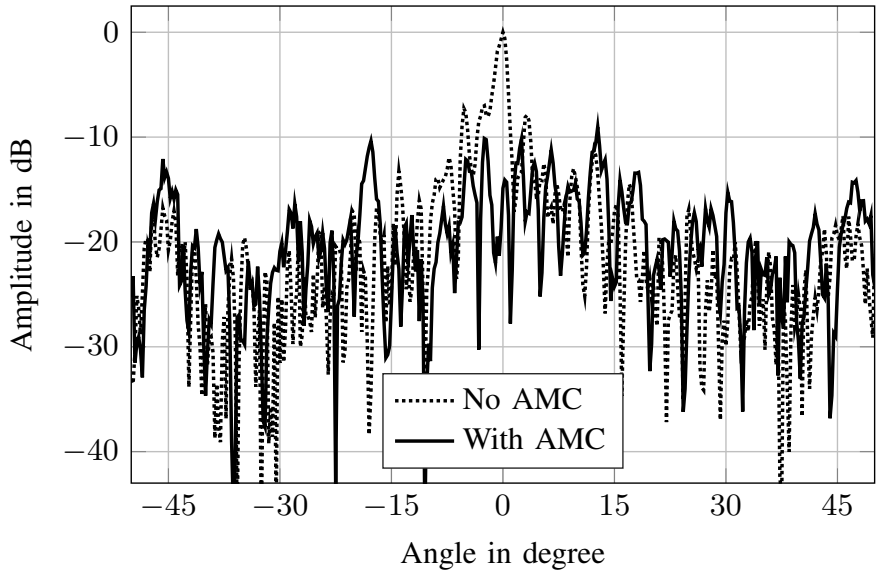

(a) Results at $77 \mathrm{GHz}$

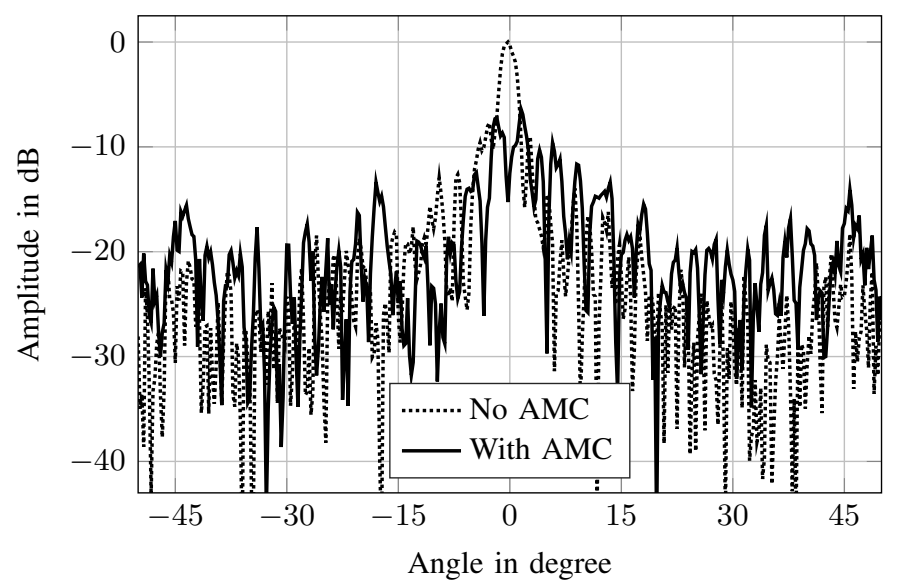

(b) Results at $79 \mathrm{GHz}$.

Fig. 5. Measured backscattering from the prototypes with and withour AMC on the azimuth plane. The curves have been normalized to an equal-size metal plate.

better visualize the multiple reflections and the effect of the low-RCS structure. The reference plane is set after a one-port response calibration at the end of the rectangular waveguide that feeds the horn antenna. The peak that represents the reflections from the bumper sample is clearly visible at the position $0.6 \mathrm{~m}$; afterward, at $0.73 \mathrm{~m}$ the backscattering from the AUT is evident. The results in the time domain confirms what has been already verified in the frequency domain: the use of the AMC permits to reduce the backscattering from the AUT. What is more interesting is to verify with this measurement setup the effect on the multiple reflections when a low-RCS antenna system is employed. At $0.85 \mathrm{~m}$ the first multiple reflection is present; as it can be seen from the significantly smaller amplitude of the peak, the low-RCS antenna system can successfully mitigate the problem of the multiple reflections between the bumper and the AUT.

\section{CONCLUSION}

This paper presented a low-RCS multiple antenna system that can be used in a mm-wave automotive radar sensor to

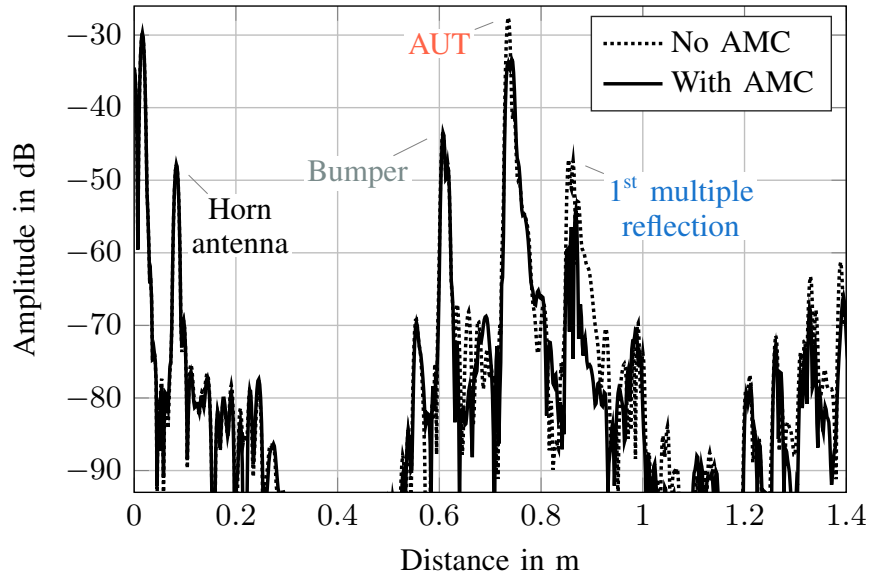

(a) Time domain measurement results.

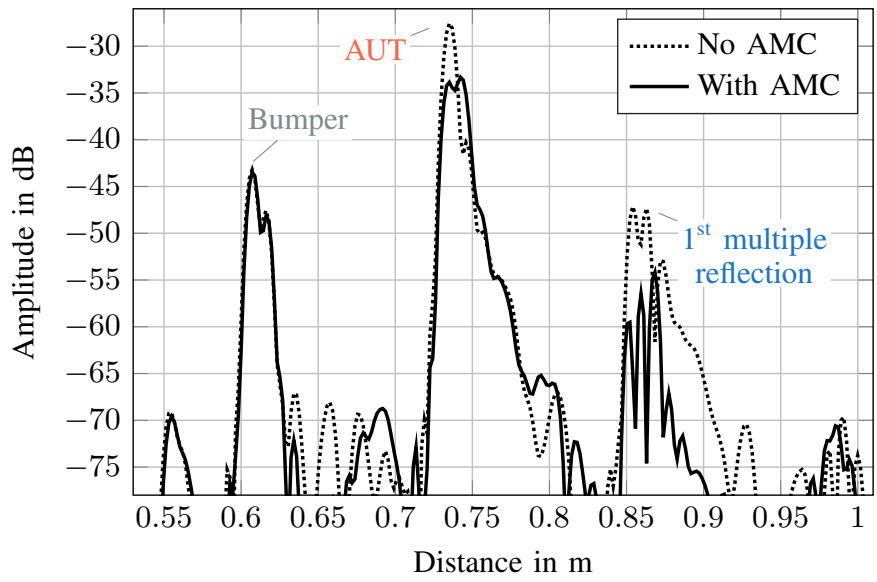

(b) Zoom into the time domain measurement results.

Fig. 6. Measurement results of the prototypes of Fig. 3 behind a sample of a car bumper. The peak that corresponds to the first multiple reflection is highlighted by the label.

mitigate multiple reflections due to presence of the vehicle bumper in the vicinity of the radar.

Based on the fundamental work presented in [6], the paper generalized the design procedure for a complex structure that mimics a MIMO antenna system typically employed in a mm-wave automotive radar. The basic idea of the design is the placement of the AMC on the same PCB of the array in order to cause a destructive interference between the wave backscattered by the array and by the AMC for a specific direction and in a narrow-band frequency range.

A simple empirical design method has been proposed, since full-wave simulations are not feasible due to the complexity of the models that have been investigated.

The fabricated antenna system prototype with reduced RCS showed $17 \mathrm{~dB}$ smaller amplitude compared to a standard array without AMC. Finally, the measurement results demonstrated also that the use of low-RCS arrays can effectively reduce the multiple reflections between the array and the bumper.

It can be concluded that the proposed solution is an effective way to mitigate multiple reflections without increa- 
sing the complexity and the costs of a mm-wave automotive radar sensor, since it is fully-compatible with standard PCB technology.

\section{REFERENCES}

[1] J. Dickmann, J. Klappstein, M. Hahn, N. Appenrodt, H. L. Bloecher, K. Werber, and A. Sailer, "Automotive Radar the Key Technology for Autonomous Driving: From Detection and Ranging to Environmental Understanding," in IEEE Radar Conf. (RadarConf), May 2016, pp. 1-6.

[2] J. Hasch, E. Topak, R. Schnabel, T. Zwick, R. Weigel, and C. Waldschmidt, "Millimeter-Wave Technology for Automotive Radar Sensors in the $77 \mathrm{GHz}$ Frequency Band," IEEE Trans. Microw. Theory Tech., vol. 60, no. 3, pp. 845-860, March 2012.

[3] R. Schnabel, D. Mittelstrab, T. Binzer, C. Waldschmidt, and R. Weigel, "Reflection, Refraction, and Self-Jamming," IEEE Microw. Magazine, vol. 13, no. 3, pp. 107-117, 2012.

[4] F. Fitzek, R. H. Rahofer, and E. M. Biebl, "Broadband Matching of HighPermittivity Coatings with Frequency Selective Surfaces," in German Microwave Conf., March 2011, pp. 1-4.

[5] C. Vasanelli, F. Boegelsack, and C. Waldschmidt, "Design and Experimental Characterization of a Surface with Low Radar Cross-Section at Millimeter-Wave Frequencies," in 46th European Microwave Conf., 2016, pp. 21-24.

[6] C. Vasanelli, F. Bögelsack, and C. Waldschmidt, "Reducing the Radar Cross-Section of Microstrip Arrays Using AMC Structures for the Vehicle Integration of Automotive Radars," IEEE Trans. Antennas Propag., vol. PP, no. 99, pp. 1-1, 2018.

[7] M. Paquay, J. C. Iriarte, I. Ederra, R. Gonzalo, and P. de Maagt, "Thin AMC Structure for Radar Cross-Section Reduction," IEEE Trans. Antennas Propag., vol. 55, no. 12, pp. 3630-3638, Dec. 2007.

[8] W. Chen, C. A. Balanis, and C. R. Birtcher, "Checkerboard EBG Surfaces for Wideband Radar Cross Section Reduction," IEEE Trans. Antennas Propag., vol. 63, no. 6, pp. 2636-2645, June 2015.

[9] C. Vasanelli, R. Batra, A. Di Serio, F. Boegelsack, and C. Waldschmidt, "Assessment of a Millimeter-Wave Antenna System for MIMO Radar Applications," IEEE Antennas Wireless Propag. Letters, vol. 16, pp. 1261-1264, 2017.

[10] E. F. Knott, J. Shaeffer, and M. T. Tuley, Radar Cross Section. Scitech Publishing, 2004.

[11] M. A. C. Niamien, S. Collardey, and K. Mahdjoubi, "Absorption and Scattering Properties of a Receiving Patch Antenna," in 9th European Conf. Antennas Propag. (EuCAP), May 2015, pp. 1-4.

[12] S. Hu, H. Chen, C. L. Law, Z. Shen, L. Zhu, W. Zhang, and W. Dou, "Backscattering Cross Section of Ultrawideband Antennas," IEEE Antennas Wireless Propag. Letters, vol. 6, pp. 70-73, 2007. 